\title{
Introductory note on the correlation between the molecular and clinical aspects of infection
}

\author{
Morris Goldner PhD ${ }^{1}$, Serge Messier DVM PhD²
}

$\mathrm{I}_{\mathrm{t}}^{\mathrm{t}}$ t has become clear that the interaction between an infectious biological agent and its recipient host was destined to address the link between the genetic potential of the pathogenic microorganism and the influence of the specific host environment. A pathogen can be thought of as if it were in a continuing process of emergence involving its DNA elements. In this sense, a pathogen may enhance or decline in virulence at any given time, but in terms of the perceived capacity for infection and the subsequent disease state, the focus becomes the in situ dependence of the pathogen. This is necessary for the development of infection into disease.

The attempt to depict the role in situ of environment is complicated, however, because conditions that influence pathogenicity are continually changing. As a result, details that are derived from clinical observations should be implemented in new experimental designs for investigations of pathogenicity. The following comments are meant to demonstrate an appreciation for, and to induce an interest in, seeking a balanced view of the aspects of infection. The comments are based on presentations from the Stanier
Society/Société Stanier Symposium, Faculté de médecine, Université de Montréal, Saint-Hyacinthe, Québec, May 11, 2001. Abstracts of the presentations follow the present commentary (pages 31 to 33 ).

Mahmoud Rouabhia ('An alternative model to elucidate the interaction between host and microorganisms in the oral cavity') wisely attempts to address the host conditions as he depicts the natural obstacles encountered by the pathogen Candida albicans. Ingeniously, he engineers an experimental oral mucosa that exposes the pathogen to the front line mucosal epithelium while it is supported by the rear guard fibroblast matrix. The influence of the epithelial layer is impressive, particularly as it relates to achieving a balance between pro- and anti-inflammatory factors that aim to deter the infection while sustaining the integrity of the host.

Terry J Beveridge ('Structural and physical properties of bacterial surfaces and their implications in pathogenicity') records the surface characteristics that offer the bacterial pathogen an inroad to its host niche. These observations reveal that the pathogen's intricate surface presents more

${ }^{1}$ Département de biologie médicale, Faculté de médecine, Université Laval, Québec, Québec; ${ }^{2}$ Département de pathologie et microbiologie, Faculté de médecine vétérinaire, Université de Montréal, Saint-Hyacinthe, Québec

Correspondence and reprints: Dr Morris Goldner, Département de biologie médicale, Faculté de médecine, Université Laval, Québec,

Québec G1K 7P4. Telephone 418-656-3351, fax 418-656-7666, e-mail mcbmgo@hermes.ulaval.ca 
than a match for the host. Through advanced microscopy, cryo-electron microscopy and atomic force microscopy, the observer learns to appreciate and gains an insight into the nanographic spatial interspersing, agile movement and guided missile activity (as in outer membrane vesicles) that make the bacterial surface components such formidable antagonists. In fact, when matching the bacterial surface with the host surface, the scale of bacterial evolution seems hardly elementary even when compared with the level of host sophistication.

Jean Barbeau ('Denture related stomatitis and the paradigm of oral biofilms') draws on nature's parallel between dental plaque and biofilms. The comparison is directed toward the classical view of dental plaque, which has driven the study of oral infections and has led to the modern day comprehension that microbial communities in nature form biofilms. Today's interest in biofilms reaches into every sphere of microbial studies, whether dealing with animate or inanimate hosts. The problems that are caused by the actions of opportunistic yeasts such as Candida albicans, in regard to dentures or dental appliances and their relationship to stomatitis, provide an excellent example of mimicry. Extensive inflammation as a desperate protective act is an added feature that concerns human hosts. By this action, the host responds to evade a potential infection, but the inflammation tends to enhance simultaneously the plaque's capability to favour the colonization status that is represented in the biofilm paradigm.

John Ruby ('The genetic identification of bacteria and their symbiotic association with oral disease'), by means of highly sophisticated genotyping models, bares the communal relationships of the oral microbiota. With these skilled techniques, he traces relationships concerning transmission and assesses pressures for clonal diversity; thus, a new, improved level of efficiency for designating whether species are predominantly important for potential pathogenic significance is being attained. These highly refined identifications offer the expectation that orderliness will be eventually achieved from the morass of microorganisms that constitute the oral flora. Given this wiser view, it is expected that the underlying principles of oral microbial community life will become apparent. Indeed, as expressed in the symbiotic relationship between the host and microbial consortiums, these principles should discern the conditions (namely health or disease) that will select for mutualism or parasitism.

James G Fox ('The epidemiology of the genus Helicobacter. A clinical and public health view on an increasingly important group of gastrointestinal pathogens') provides a real eye-opener for the story of the Helicobacter species. When it comes to the microbial world, the drama that has unfolded as to the cause and effect relationship between Helicobacter pylori and stomach ulcers in humans has, indeed, been a lesson not to be easily forgotten. Through recent studies, this world, as it pertains to the Helicobacter species, has expanded - not only through the connection of Helicobacter pylori to stomach ulcer, but also because an increasing number of Helicobacter species are found in the intestinal tracts of humans as well as in a variety of animals. This has been further demonstrated in association with hepatitis, resulting in both infection and concern for carcinomas in terms of zoonotic implications. These findings have opened up an unexpected interest in the distribution and transmission of the various species, and, more directly, in treatment through the use of antimicrobial agents. Evidently, this matter will require greater research efforts to provide explanations that lead to correlations between molecular and clinical aspects of infection.

Paul S Hoffman ('Functional genomic studies of Helicobacter pylori: Applications to biology, therapeutics and vaccines') is ready, as if made to order, to answer the plea for understanding the molecular aspects of the infection, and to search for future correlations between the molecular and clinical aspects of Helicobacter species infection. The most recent techniques for comparative genomic studies revealed the uniqueness, and perhaps the unexpected resilience, of this remarkable pathogenic species, which has become so important in such a short time. By using the knowledge gained by such sophisticated genomic studies as gene subtraction and protein-to-protein interaction mapping, the functional genomic studies, drawn from the powerful tool of bio-informatics, permitted the discovery of exploitable surprises, particularly with energy metabolism genes. It appears that Helicobacter pylori may be rather selfreliant with respect to its environment. Watch for it, there is more to come!

Patrick Boerlin ('Epidemiology of antibiotic resistance in bacteria from swine') drives home the reality of resistance with the extensive use of antibiotics. In his porcine studies, this resistance is poignantly demonstrated as high among food animals, particularly in view of the international spread of resistance and the recent ban on antimicrobial growth promoter. The patterns of resistance that are illustrated under these circumstances place the culpability on the transfer of genes. These situations are changing the normal flora, as seen in the diversity of resistant strains that are repeatedly recovered, as well as in the clear difference that results from the presence and absence of selecting agents. This enormous study, involving resistant enterococci on the one hand and Escherichia coli on the other, demonstrates significantly that the normal flora are a large reservoir that carry the integrase gene, leaving no doubt as to the molecular consequences of resistance gene distribution. The deleterious effects are evidenced by clinical infections in domestic animals and in their transfer to humans.

Marcel A Behr ('Molecular epidemiology of TB in humans and livestock') eloquently educates us about the molecular typing methods that have been developed to answer epidemiological questions about Mycobacterium tuberculosis. These techniques pertain especially to genomic insertion sequences, which have, in turn, been the mainstay of molecular fingerprinting of Mycobacteria species.

The limitations of earlier typing methods resulted in the serious need for new methods. The molecular techniques 
used in the study of the epidemiology of tuberculosis have provided greater integration in disease control, including epizoological issues, and have increased our ability to identify clinical isolates with unusual phenotypic properties. These outstanding efforts have increased epidemiologists' means for following the 'more virulent' predominating strains. The advanced methods have also been applied to study naturally attenuated mutants such as those that are used in Bacille Calmette-Guérin (BCG) vaccines. The gain has been in exerting better disease control and, importantly, in obtaining information about gene content; thus, the search for the correlation between molecular character and clinical phenotype becomes ever more feasible and valuable.

How do we emphasize the correlation between the molecular findings and the clinical picture? It is known that pathogenic microorganisms present themselves as overt, insidious, emerging or re-emerging, and it is understood that this knowledge must extend from fundamental processes to clinical expression. To the extent that it is feasible, basic and clinical pathogenicity research will come together in close collaboration to address the same problems and to closely associate the potentiality and reality of the pathogen. The most usefulness will likely come when the molecular studies are tempered by the clinical picture. This interest underlines the necessity for concern regarding both the genome and the environment.

ACKNOWLEDGEMENTS: The helpful criticism of our colleague, Raina Fyson, Ottawa, is gratefully acknowledged. 


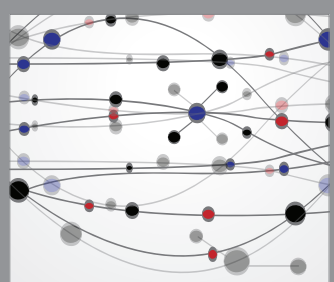

The Scientific World Journal
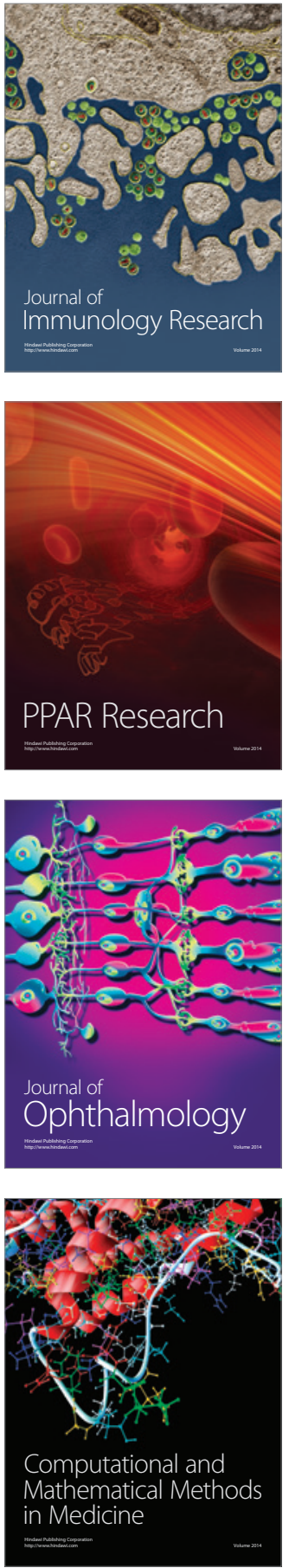

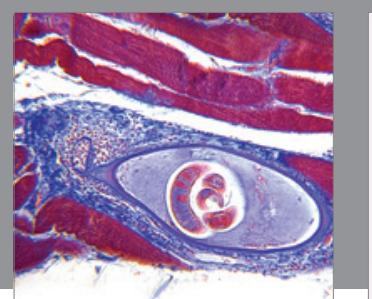

Gastroenterology Research and Practice

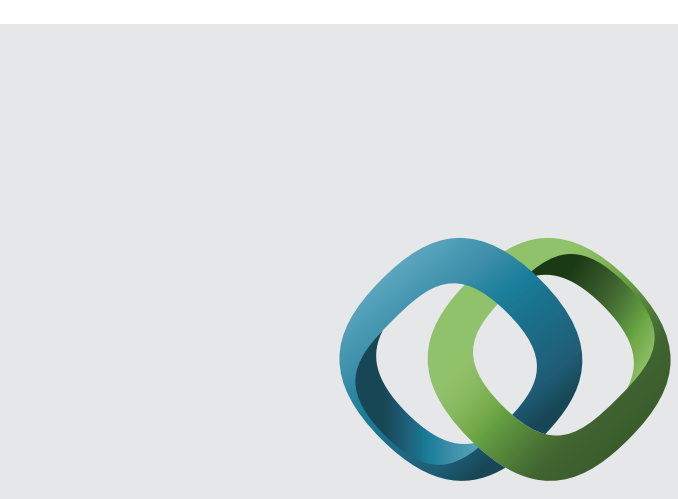

\section{Hindawi}

Submit your manuscripts at

http://www.hindawi.com
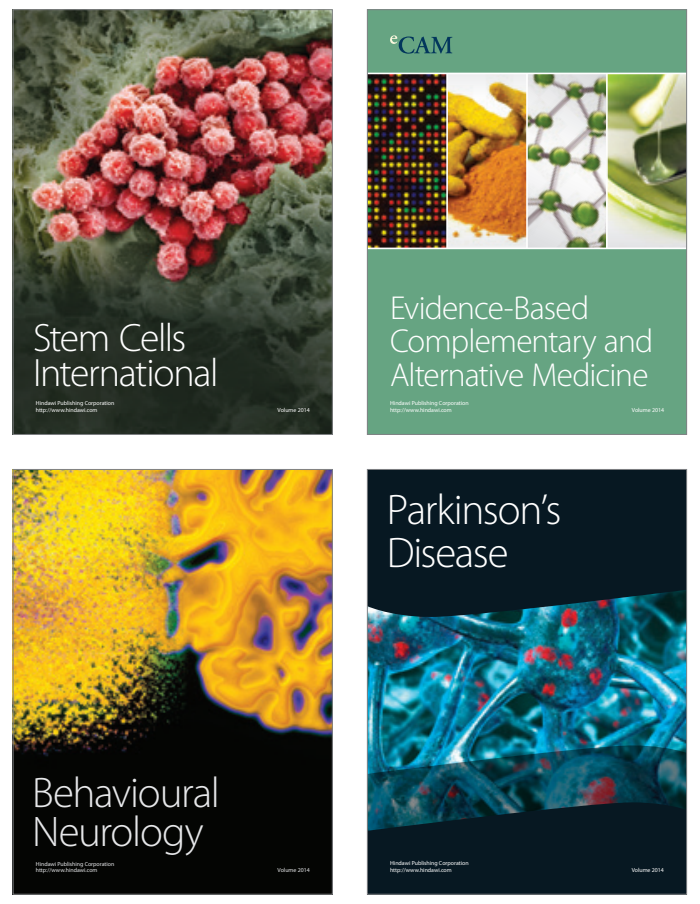
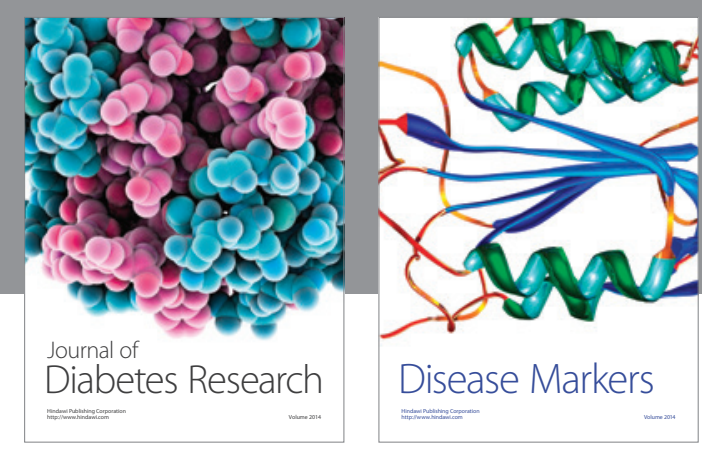

Disease Markers
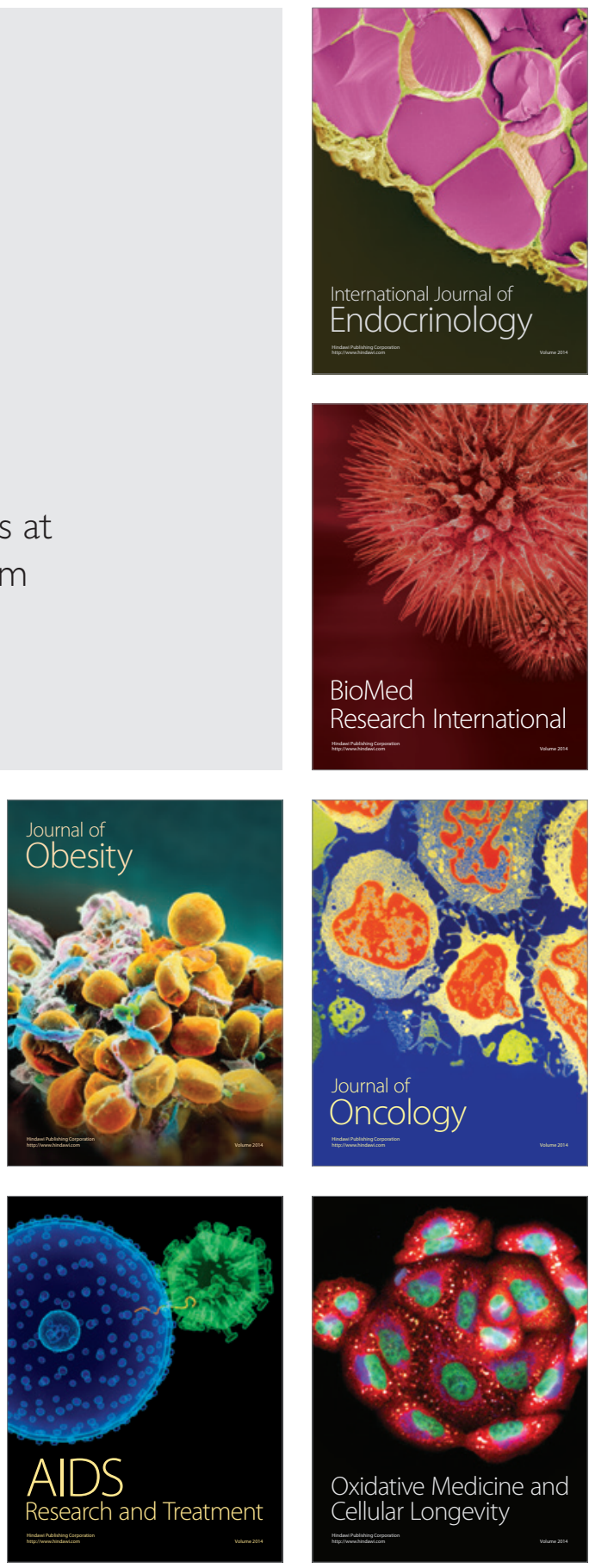